\title{
GESNERUS
}

Vierteljahrsschrift für Geschichte der Medizin und der

Naturwissenschaften

Revue trimestrielle d'histoire de la médecine

Jahrgang - Volume - $1 \quad$ 1944 Heft - Fasc. -4

\section{Un Régime pour garder santé}

donné au duc de Savoie par un gentilhomme vaudois

il y a cinq cents ans

Le donateur - Le prince — L'auteur — Le livre — Le sort du volume

Annexe: Liste de Régimes

\section{A Arnold C. Klebs. 17 mars 1940}

\section{Le donateur}

Le Régime pour garder santé de corps et d'âme et parvenir à belle et plaisant vieillesse a été préparé, comme la dédicace nous en informe, «à la procuration et diligence de noble home Françoys Rossyn seigneur d'Aleman», désireux d'en faire hommage à son souverain le duc de Savoie.

Lorsque la notice ${ }^{1}$ où ce volume était mentionné, et qui reproduisait en entier les dix lignes du préambule, tomba sous les yeux de M. Maxime Reymond, directeur des Archives cantonales vaudoises, l'identité du donateur cessa aussitôt d'être un mystère. ${ }^{2}$ En effet les Russin sont seigneurs d'Allaman, au Pays de Vaud, à partir de 1437; il ne restait donc qu'à chercher les François de la famille et, s'il s'en trouvait plusieurs,

1 Alphonse Bayot, Les manuscrits de provenance savoisienne dans la Bibliothèque de Bourgogne [à Bruxelles]; Mém. et Doc. publiés par la Soc. sav. d'hist. et d'archéologie, Chambéry, XLVII, 1909, p. 305-410. - Sur le texte d'Héronchel, p. 325-327; cote du ms, 11198.

2 Maxime Reymond, Livre donné par un Lausannois au duc de Savoie. Revue Historique Vaudoise (RHV) 1912, p. 254-255. 
décider lequel avait pris cette initiative. Si, aujourd'hui encore, certains points de la généalogie des Russin sont insuffisamment fixés, il ne peut subsister de doute que notre donateur est François II, fils de François I.

Le premier de ces François était passé de Savoie au Pays de Vaud vers 1380. Originaire d'Evian, ${ }^{3}$ donzel, il était juriste et fit une belle carrière, qui lui valut honneurs et richesses; châtelain de Morges de 1384 à 1387, il sera bailli épiscopal, c'est à dire juge suprême établi par l'évêque, de 1408 à 1416; seigneur de Corsy sur Lutry, propriétaire de plusieurs maisons et terrains. Etant mort avant 1427, il ne peut être notre donateur.

C'est son fils François II, châtelain de Nyon, qui acquit Allaman, le 17 décembre 1437, pour 3000 florins petit poids. Il meurt entre 1461 et 1463, laissant la seigneurie à son fils Jaques. Le don du Régime se place ainsi entre 1437 et 1463 .

Allaman resta dans la famille jusqu'en 1546, où le dernier des Russin, Jean, accablé de dettes, dut le vendre. Entre temps les Bernois avaient profité d'une expédition au secours de Genève pour brûler le château en 1530. Pendant tout le $15 \mathrm{e}$ siècle, sans jamais être nombreux, trois générations de Russin possédèrent en outre la belle seigneurie de Bottens et de nombreux fiefs; la dernière des propriétés de la famille dut être aliénée en $1555 .^{4}$

En même temps qu'ils poursuivent leur carrière dans le Pays de Vaud, les Russin restent attachés à la cour de Savoie. Précisément dans les années 1434 à 1436 qui précèdent l'acquisition de la seigneurie d'Allaman, François et Jaques Russin font partie de la suite de Louis de Savoie. François est chargé de préparer en 1436 une chapelle à Thonon, pour la

3 Russin est un petit village, alors savoyard, genevois depuis 1746, à quelques kilomètres de Genève, rive droite du Rhône. Sans doute la famille venait de là; mais lorsque François I apparaît dans nos documents il est toujours dit d'Evian.

${ }^{4}$ Sur les Russin dans le Pays de Vaud, voir, entre autres, outre l'article cité de M. Reymond: Dictionnaire hist. et géogr. du canton de Vaùd par E. Mottaz, s. v. Allaman, Bottens, Bussigny, Corsy s. Lutry, Morrens, Prilly. - Ch. Morton, Les nobles de Russin au Pays de Vaud, Arch. héraldiques Suisses, 1929, p. 176-181. - D.-L. Galbreath, Armorial Vaudois, II, 612, avec fig. 2016, 2017, et pl. L. - Si le tableau d'ensemble offert par ces imprimés est exact dans les grandes lignes, le recours aux pièces d'archives fait pourtant constater nombre de confusions et d'erreurs, portant sur personnages ou dates; ce n'est pas ici le lieu où les relever. Une des maisons des Russin à Lausanne, angle rue de Bourg place St François, reçut longtemps le Plaît Général; une autre, à la Madeleine, hébergeait en 1440 une école de la ville. 
venue de Yolande de France; surtout, il dirige les installations nécessaires pour la gésine de la duchesse Anne; le compte détaillé est conservé, ainsi que pour le baptême de l'enfant, le futur Amédée IX, né le ler février 1435; le confesseur de la princesse dut venir exprès de Bâle. En retour de ces bons services, Anne de Savoie sera quelques mois plus tard, à Evian, marraine d'un enfant de François Russin. ${ }^{5}$

\section{Le prince}

C'est au «très excellent prince, le duc de Savoie», qu'est dédié notre Régime. Pareille épithète, dans une dédicace, ne signifie guère. De fait, Louis, - car c'est de lui qu'il s'agit, très probablement - est loin de briller dans la série des chefs du pays; il n'est pas, comme son père, une personnalité.

Le père vivait encore lorsque Louis prit la charge du duché; ou plutôt la reçut, à titre de lieutenant-général, car Amédée VIII, à peine entré dans la cinquantaine, en pleine possession de tous ses moyens, décida (1434) de renoncer, au moins partiellement, au pouvoir et de consacrer à la retraite la fin de sa vie. Non point pour s'y livrer à la paresse ou à des jouissances grossières; au contraire, il se proposait d'y cultiver les lettres, les arts, la sagesse, dans le cadre d'une vie religieuse. Il fonda dans ce but un ordre de Saint Maurice, groupant six seigneurs lettrés, dont il serait le chef, dans l'ermitage de Ripaille. ${ }^{6}$ Le choix qu'il fit de ses compagnons est significatif, à juger par les deux que voici. Henri de Colombier, grand seigneur du Pays de Vaud, avait été chancelier de Savoie, capitaine du Piémont, conseiller ducal, avait combattu cinquante ans sous la bannière de Savoie, type du gentilhomme cultivé et riche d'expérience. ${ }^{7}$ Martin Le Franc lui fait pendant comme homme d'église lettré; il sera prévôt du chapitre de Lausanne, protonotaire apostolique, secrétaire de deux papes, et surtout auteur de deux grands ouvrages, en vers (1440-42) et

5 Max Bruchet, Le château de Ripaille, Paris 1907; p. 145 n. 6; p. 480-483, Preuve LXI; p. 486, Preuve LXIII; p. 509, Preuve LXX. Je ne suis, il est vrai, pas certain que ce François soit identique avec le futur seigneur d'Allaman; la naissance d'un enfant à cette date s'accorde difficilement avec ce que l'on croit savoir du mariage de notre François II, qui avait épousé avant 1402 Froa de Colombier, encore vivante en 1452.

${ }^{6}$ Voir Bruchet, op. cit.

7 E. Dupraz, Relations des seigneurs de Colombier avec l'Abbaye de Montheron $\mathrm{R} \mathrm{H} \mathrm{V}$ 1909 p. 37 ss. 
en prose mêlée de vers (1447-49), trop longs sans doute, mais pleins de verve et de sagesse. ${ }^{8}$

Au moment où Amédée s'apprêtait à prendre une demi-retraite, il pouvait se rendre le témoignage qu'il l'avait méritée; il avait fort agrandi l'étendue de ses territoires; de comté, la Savoie était devenue duché (1416); il s'était montré aussi habile négociateur que bon militaire. Pourtant, ses beaux plans ne se réalisèrent pas autant qu'il l'eût désiré; car le Concile de Bâle, désireux de faire progresser la réforme de l'Eglise, s'adressa à l'ermite de Ripaille pour lui offrir la tiare (1439). La chrétienté resta pourtant divisée, les deux papes opposés l'un à l'autre; par gain de paix, Félix V finit par abdiquer à Lausanne le pouvoir religieux suprême (1449), comme il avait, quinze ans plus tôt, commencé à se retirer de l'arène politique.

Comparé à ce père, qui a de la grandeur, Louis fait petite figure. Il avait épousé une femme trop belle, capricieuse, impérieuse - qui lui donna d'ailleurs au moins quinze si ce n'est dix-sept enfants, - Anne de Lusignan, de la famille des rois de Chypre; elle apporta dans la Savoie montagneuse les goûts de luxe et les habitudes de favoritisme d'une demi orientale; d'où désordre dans l'état sous un prince mou, indolent, indécis, incapable. Les trente années de gouvernement de Louis († 1465) ne furent heureuses, ni pour lui ni pour le pays. Il faut reconnaître qu'il ne fut pour rien dans le choix de son épouse. Son père seul rechercha pour lui cette princesse lointaine dont l'entrée dans sa famille devait rehausser, pensait-il, le prestige de la maison de Savoie. Même les plus sages des hommes font de ces calculs trompeurs; et ce n'est pas la seule fois que le mirage oriental fut néfaste à la dynastie.

A sa mort, en 1465, Louis avait cinquante deux ans. Depuis longtemps il souffrait de la goutte. Peut-être en était-il déjà atteint lorsque François de Russin, dans son désir de le soulager, lui donna le Régime. Je ne sache pas que le duc se soit livré à des écarts susceptibles d'aggraver le mal; cependant, comme Klebs me l'a fait remarquer, il était gourmand; peut-être gros mangeur. Un de ses médecins, Pantaléon de Confienza, dans son délicieux petit traité des fromages, Summa lacticiniorum, où il passe en revue tous les fromages connus de lui, nous confie que le duc en était un fin connaisseur. Au cours de plusieurs mois qu'ils passèrent ensemble à Paris, nos deux experts furent si satisfaits des Brie qu'ils y con-

8 Arthur Piaget, Martin Le Franc, prévôt de Lausanne; Genève 1888. 
sommèrent, qu'ils étaient prêts à accorder à cette marque la palme sur toutes les autres; et cela, malgré les excellents produits fournis par la Savoie elle-même. ${ }^{9}$ Peut-être est-ce pour avoir trop aimé sa femme et le Brie que le duc Louis eut besoin d'un Régime!

Le don du Régime est, nous l'avons vu, postérieur à 1437. Un autre motif, que nous indiquerons plus loin, nous engage même à le retarder jusque vers 1450 environ. Dernière raison de nous faire admettre que, entre Amédée et Louis, c'est le fils auquel il a été destiné, et non le père.

\section{L'auteur}

L'auteur a l'amabilité de donner son nom, il signe Heronchel. C'est à peu près tout ce que nous savons de lui; autant dire: rien. Aucun recueil bibliographique ne lui a fait l'honneur de le noter. ${ }^{10}$

Pourtant, tout à la fin de son Régime, une confidence lui échappe: «en notre deux pays de Normandie», dit-il. Des recherches rendues sommaires par la dureté des temps, faites à Rouen par Mlle Jeanne Dupic, bibliothécaire, ancienne élève de l'Ecole des Chartes, ont du moins établi qu'il existe à quelques lieues au nord de Rouen un village Héronchelles et qu'au quinzième siècle, des gens de l'endroit sont désignés comme: de Heronchel. ${ }^{11}$ Confirmation bien sommaire, pourtant précieuse.

D'autre part, la Bibliothèque de Lausanne possède deux volumes manuscrits d'une grande Bible écrite par le genevois Jean Servion. Des prologues précèdent un certain nombre de livres; trois d'entre eux ont été traduits du latin, de Saint Augustin, par «mâ̂tre Pierre Aronchel». ${ }^{12}$ Comme Servion écrit à Genève entre 1455 et 1462, et que François de Russin d'Allaman fait don du Régime dans les années 1437-1463, le

9 Summa lacticiniorum, II, 11. - Imprimé pour la première fois en 1477 ou peu après, a eu encore plusieurs éd. au 16e siècle, 1508, 1517, 1525, 1528, 1568, d'autres peut-être. Pour l'éd. incunable, v. Arnold C. Klebs, Incunabula scientifica et medica, Short Title List, 1938, no 719.1. L'éd. que j'ai vue, de la Biblioth. Klebs, est de 1508, Pavie, in folio, gothique, avec le Pillularium du même auteur. - Sur Pantaléon, v. E. Wickersheimer, Dictionnaire biographique des médecins en France au moyen âge, 591. Docteur de Pavie en 1440, il sera, pour finir, professeur à cette université, 1492-96. De 1472 à 1479 il fut médecin de Louis XI.

${ }_{10}$ E. Wickersheimer l'accueille dans son Dictionnaire, op. cit., p. 294, d'après le mémoire de Bayot cité ici note 1.

11 Lettre de Melle J. Dupic, 17 janv. 1940.

12 Denise Werner, Jean Servion, figure genevoise du 15e siècle; R. d'hist. suisse, 1934, p. 204-237; en particulier, p. 222, 225. 
rapprochement des noms, des dates, des régions, donne naturellement à penser que Héronchel et Pierre Aronchel ne font qu'un. Malheureusement, ni le professeur Piaget, ni Mlle Werner n'ont rencontré nulle part le moindre renseignement sur Pierre Aronchel, en dehors de sa participation à l'œuvre de Servion. ${ }^{13}$

Peut-être un autre contact encore peut-il être supposé, et cette fois entre Héronchel et Martin Le Franc, le prélat littérateur, commensal d'Amédée VIII, secrétaire de Félix V. Le Franc a, lui aussi, traduit un des prologues de la Bible Servion; et lui aussi est originaire de Normandie. ${ }^{14}$ N'est-il pas permis de se figurer qu'il a pu protéger son compatriote, le mettre en relation avec les lettrés du Pays de Vaud ou de Genève, avec l'entourage de la cour de Savoie?

Resterait à trouver des indices de la présence de Héronchel dans la suite du duc Louis. Jusqu'ici nous n'en avons rencontré aucun; tout comme les archives de Genève et de Lausanne sont muettes à ce sujet.

Nous pouvons enfin, pendant que nous nous abandonnons au petit jeu des conjectures, envisager une rencontre entre le duc, son médecin Pantaléon, en quête de fromages de Normandie, et le clerc lettré Héronchel?

Quoi qu'il en soit de sa personne, nous ne devons pas tenir pour un médecin Héronchel, signataire du Régime pour garder santé. C'est un homme qui sait suffisamment de latin pour mettre en français les textes qu'on lui propose, qu'il s'agisse de commentaires bibliques ou d'un traité d'hygiène. C'est ce que nous allons voir de plus près. D'autre part, si, comme nous le pensons, Héronchel et Pierre Aronchel ne font qu'un, cela nous engage à placer le don du Régime, non point vers le début du règne de Louis, mais aux environs des années où Aronchel fournit à Servion ses traductions. Le Régime serait ainsi de 1450 à 1460 , plutôt que de 1440 à 1450 .

\section{Le livre}

Maître Héronchel a pour le lecteur une deuxième attention; il confesse d'emblée que son Régime pour garder santé de corps et d'âme et parvenir à belle et plaisant vieillesse, n'a rien d'original; qu'il est «extrait et translaté» de la Discipline de médecine.

13 Leurs lettres, 19 mars 1940, 30 nov. 1934, 2 août 1940.

14 Le Franc était né «en la doulce conté d'Aumalle», nous apprend A. Piaget, op. cit., p. 12. Maître Pierre Aronchel est mentionné, p. 19, à propos des prologues fournis à Servion. 
Aveu précieux; si seulement nous connaissions cette Discipline! Mais ce titre ne se rencontre nulle part. En sorte qu'il ne restait, apparemment, qu’à prendre toute la série des Régimes de santé antérieurs au milieu du $15 \mathrm{e}$ siècle, pour voir, si possible, auquel notre traducteur avait emprunté son texte. Une heureuse étoile veillait apparemment sur cette entreprise, qui risquait de mener loin: le premier des ouvrages que nous avons examinés se trouva être l'original même; c'est le Regimen sanitatis ad inclitum regem Aragonum directum et ordinatum, dont l'auteur n'est autre que l'illustre médecin, alchimiste, ambassadeur de rois, confident de papes et pourtant théologien hérétique, Arnauld de Villeneuve. La date exacte de sa rédaction n'est pas connue; elle ne s'écarte guère de 1300 , avec une marge d'une dizaine d'années en chaque sens (Arnauld $† 1311$ ).

Le traducteur travaille, en somme, honnêtement sur son texte; il lui arrive de sauter par ci par là une phrase; il aime allonger les énumérations, il penche au délayage alors qu'Arnauld est bref et «matter of fact»; il marque son indépendance en coupant l'ouvrage en quatre parties et en multipliant les chapitres; il supprime le dernier, qui traitait en détail des hémorrhoïdes, et dont on peut d'ailleurs se demander s'il n'est pas une notice indépendante qui aurait été rajoutée après coup au corps de louvrage. Ce ne sont là que des modifications tout extérieures; pour le surplus, Héronchel avance mot à mot, ligne à ligne, page à page.

Il est honnête, c'est à dire qu'il fait de son mieux; il n'est jamais brillant et même pas toujours exact, en particulier dans les translations de termes médicaux. On le saisit parfois en flagrant délit d'ignorance. Il n'est pas correct de rendre screatus in pectore par salive en la fourcelle (23), ni supercalescere par échauffer, au lieu de surchauffer $(23 \mathrm{v})$, ni porcellus par porc (47). Il a des distractions, des négligences, des contresens, qui révèlent l'homme qui ne prend pas la peine de se relire; il remplace ludus lancealis par la danse (10); placeant par préparent (37 v); congruunt par restreignent $(30 \mathrm{v})$; ventositas par viscosité (41); de pullis gallinarum devient de la pulpe de gelines $(47 \mathrm{v})$; et le comble du quiproquo est un brouet de congre (52) qui vient remplacer l'avis tout général qu'un brouet conviendrait dans tel cas, brodium congruit! Il serait facile de cueillir une vingtaine de bévues de ce genre.

Les adjonctions qu'il se permet ne nous apprennent pas grand chose sur lui, en dehors de sa prétention à grossir les listes d'objets données par Arnauld, surtout pour les aliments. Par exemple, Arnauld mentionnant "persicae et antepersicae», Héronchel en tire (34 et 34v) des «pesches, 
persiques, percopes et propersiques»; ce qui ne contribue pas précisément à nous éclairer sur la distinction que le Catalan entendait faire. Il se pique de qulque érudition, amenant à la barre une fois Aristote (25 v), une fois Caton (27); il raconte qu'on peut traiter le vin par la glaire d'œuf (19); à une place où Arnauld nomme le diaphragme sans le décrire, Héronchel se décide à le faire (35 v); "le dyafragme, qui est en la fourcelle, une pel composée de trois substances panniculères et devise les membres nutritifs des esperituelz». Probablement le morceau dont il était le plus fier est celui qu'il introduit (13 v) à l'endroit où Arnauld définit la faim, le héraut que la nature nous envoie pour nous avertir de manger. Sur quoi Héronchel ne peut se retenir de se lancer dans une pesante allégorie d'une page, sur «le herauld de nature transmis devant pour annuncier le payement du tribu de viande qui est la droicte rente du corps, car s'il n'estoit payé guerre seroit ouverte entre le ventre et nature»... Et cette extravagance finit par un appel aux «loys civiles [qui] ont mis bride à ceulx qui sont immodérés créditeurs»... Aberration révélatrice du goût de l'époque.

Le Regimen ad regem Aragonum a connu un succès durable; à preuve qu'il en existe sept éditions incunables; ${ }^{15}$ l'une, séparée, celle même dont le Dr Klebs m'a prêté une photocopie, les autres accompagnant le Regimen sanitatis Salernitanum; outre les nombreuses éditions des Oeuvres d'Arnauld d'après le texte de Thomas Murchi, au moins huit au $16^{\mathrm{e}}$ siècle.

Malgré quoi, chose curieuse, il ne semble pas qu'une traduction française en existe, en dehors de celle de Héronchel; du moins n'en ai-je trouvé aucune mention dans les listes que j'ai pu consulter. Il en existe une espagnole et au moins une hébraïque. ${ }^{16}$

Telle est la part du traducteur dans la préparation de notre texte. Que savons-nous des intentions de l'auteur, Arnauld le Catalan?

15 Klebs, op. cit., 99.1 et $829.1-6$.

16 Cf. Hauréau, Arnauld de Villeneuve, dans Hist. littéraire de la France, XXVIII, 1881, p. 26-126; sur le Régime au roi d'Aragon p. 55 s. - Le texte Héronchel n'a aucun lien avec un autre ouvrage d'Arnauld dont plusieurs éd. françaises ont paru au début du 16e siècle, le Régime très utile et tres proufitable pour conserver la santé du corps humain. Mentionné par Wickersheimer, op. cit. 49, mais non par Hauréau, ce Régime-là est la version française du Commentaire d'Arnauld sur le Regimen Salernitanum (Klebs, no 830.1; texte latin, commentaire français). 
Son livre est loin d'être le premier, ou le dernier, d'une série, fort longue au contraire, qui connut la vogue pendant plus de trois siècles. Essayons d'aligner les principaux de ces ouvrages. Les Incunabula de Klebs nous fourniront d'abord ceux que les imprimeurs du $15^{\mathrm{e}}$ siècle firent passer par leurs presses; ils sont une quinzaine. Ajoutons les écrits restés manuscrits, les imprimés postérieurs à 1500 - dont les textes peuvent remonter plus ou moins haut - nous dépassons le double. ${ }^{17}$

Cela, sans compter ceux qui s'adressent à certaines classes de la population, pauvres, paysans, soldats, marins, voyageurs, etc.; ou aux divers âges; ou qui suivent le cycle des saisons ou visent les fléaux épidémiques; sans compter les diètes ... Sans compter les innombrables éditions du Régime de Salerne, qui lui aussi nous ramènerait à Arnauld mais auquel sa forme différente assura un sort différent. Sans compter, enfin, les divers Mirabilia mundi, Secreta naturae, Thesaurus pauperum, etc., qui souvent cultivent un terrain bien proche du nôtre, quand il ne se confond pas avec lui.

Arnauld lui-même a multiplié ses conseils d'hygiène, car en dehors du Régime au roi d'Aragon et de ses Commentaires à Salerne il a encore écrit: un «Régime» pour le pape Clément $\mathrm{V}$ (mais qui ne contient que des recettes); un traité De conservanda juventute et retardanda seneciute; un régime des vieillards et un autre pour les militaires, qui ont tous été imprimés; auxquels s'ajoutent deux manuscrits encore inédits, de conservatione humane vite, et de dieta. ${ }^{18}$

Laissant de côté toute cette production, revenons au Régime au roi d'Aragon. Arnauld y expose brièvement, à l'usage du prince, les règles générales d'une vie hygiénique, dans le cadre coutumier des six choses habituellement dites non naturelles; commençant par l'air, le mouvement et le repos, les soins de propreté, le sommeil et la veille, les passions de l'âme; pour s'étendre surtout sur le boire et le manger. S'il s'adresse au roi, c'est d'abord parce que sa personne est la plus précieuse de l'état; mais il prévoit en outre que l'exemple venu de haut sera imité d'abord dans son entourage immédiat et ensuite par degrés dans des couches de plus en plus étendues de la population. Que l'élite montre la voie, les autres suivront. Ainsi faisant, il se trouve d'accord avec bon nombre des auteurs qui figurent sur notre liste; la plupart dédiant leur œuvre à quel-

\footnotetext{
17 Voir cette liste à la fin, en annexe.

18 Cf. les ouvr. cités de Hauréau et de Wickersheimer, à la note 16 ci-dessus.
} 
que grand seigneur à défaut d'un roi; façon, aussi, de mieux appâter le lecteur!

Quant aux relations qui unissent entre eux ces Régimes dus à tant d'auteurs divers, je les connais beaucoup trop peu pour m'être fait une opinion sur leur filiation spirituelle possible. Sudhoff ${ }^{19}$ se disait convaincu qu'ils dérivent tous de la Lettre d'Aristote qui porte sur notre liste le No 1 . Il se peut que ce soit le cas pour la plupart; pour en juger, il faudrait en examiner un grand nombre; or je n'en ai guère vu que le quart. Arnauld, pour sa part, dans ses conseils au roi d'Aragon, reste relativement indépendant. Le cas de rencontres fortuites ou nécessaires entre ces écrivains doit aussi se présenter assez souvent, étant donné l'identité du sujet. Il va d'ailleurs de soi que même si la Lettre d'Aristote, fabriquée par quelques compilateurs arabisants, a donné le branle au mouvement, le contenu de toute cette littérature est grec, et à travers Avicenne, Isaac et consorts, vient en réalité d'Oribase, de Galien, des hippocratiques. ${ }^{20}$

\section{Le sort du volume}

Le livre donné par François de Russin semble avoir été apprécié par le prince; en a-t-il appliqué les règles — c'est une autre question. Autant que nous pouvons le savoir, Amédée VIII n'avait pas d'ouvrages de médecine dans sa bibliothèque. ${ }^{21}$ L'inventaire de la bibliothèque ducale dressé

19 Meyer-Steinegg und Sudhoff, Geschichte der Medizin, 3e éd. 1928, p. 234.

20 R. Pépin, dans l'éd. qu'il a donnée en 1911, avec Landouzy, du Regime du corps de maître Aldebrandin de Sienne, a établi comme ses sources: Avicenne, Hali Abbas, Johannitius (Honain ben Ishak), Razes, Constantin, Isaac le Juif; c'est à travers eux seulement que l'auteur entre en contact avec Ypocras, Aristotele et Gallien. - J. Brinkmann, Die apokryphen Gesundheitsregeln des Aristoteles für Alexander den Großen, diss. Leipzig 1914, constate des emprunts à Oribase. - Telles règles de notre Arnauld sur l'exercice (f. 4) concordent avec celles de Celse I, 2.

21 Voir l'essai de reconstitution de celle-ci, tenté par Bruchet, op. cit., 159, 372-375. Elle contient bien un De regimine principum; Bruchet le tient pour un traité de philosophie morale. Ce même titre a cependant été aussi donné, par exemple, à des extraits des Secreta secretorum du Pseudo Aristote, (Klebs, Incunabula 96.01), sur lesquels v. notre liste de Régimes, no 1. Nous ne pouvons en tous cas pas accepter l'interprétation de Bruchet touchant deux ex., dont un en italien, du livre «de Mandevie», où il voit la chirurgie de Mondeville. Celle-ci n'a jamais été traduite en italien. Il s'agit des Voyages du célèbre sir John de Mandeville ou Mandevie, qui faisaient fureur dans le monde lettré. 
en $1479^{22}$ mentionne par contre, au No 58, «vng petit liure en papier, appellé Heronchel»; celui de 1498 l'inscrit, No 111, avec un peu plus de détails sur son apparence et avec son titre, mais sans le nom de l'auteur. ${ }^{23}$ Il est à ce moment relié en parchemin collé sur papier. Il n'est pas non plus resté seul de son espèce. En 1479 il n'a encore qu'un seul compagnon, un «Erbolaire», couvert de bois ${ }^{24}$ en 1498 j'en vois, autant qu'on peut s'en rendre compte sur la seule apparence des titres, peut-être quatre ou $\operatorname{cinq} \cdot{ }^{25}$

Après quoi, Philibert II le Beau, deux fois marié, étant mort en 1504, sans enfant, sa veuve, Marguerite d'Autriche, quittant la Savoie pour les Pays Bas qu'elle va gouverner, emporte le Régime pour garder santé. C'est ainsi que la Bibliothèque royale de Bruxelles le possède aujourd'hui; relié de velours vert portant l'ex-libris de Marie de Hongrie et même l'estampille rouge, aux faisceaux, de la Bibliothèque Nationale, car à l'époque révolutionnaire il dut faire un séjour à Paris.

Résumons notre récolte. Aux environs de 1450, François II de Russin, seigneur d'Allaman, donne à son «très excellent prince», le duc Louis de Savoie, le Régime pour garder santé de corps et d'âme et parvenir à belle et plaisant vieillesse. C'est la traduction du Regimen sanitatis ad inclitum regem Aragonum, écrit par Arnauld de Villeneuve un siècle et demi plus tôt; autant que nous le sachions, le seul texte français de cette œuvre. Celui qui l'a «extrait et translaté» signe Heronchel; il est normand et très vraisemblablement identique à Pierre Aronchel, qui à la même époque traduit pour le genevois Jean Servion des prologues - commentaires à divers livres de la Bible.

22 Dans Miscellanea di storia italiana, XXII Turin 1884, p. 348-362. L'éditeur se demande d'abord, p. 355, no 58, s'il s'agirait du roman d'Hercules; puis, dans une correction, p. 376, met déjà ce nom en rapport avec celui du traducteur de prologues de la Bible Servion, et conclut qu'il s'agit ici aussi de ces mêmes prologues.

23 Ibid. p. 49 no 111; avec renvoi à une mention incomplête dans la Bibliothèque protypographique de Barrois, qui conduit l'éditeur à supposer que François Rossyn serait un médecin Rossi, de Novare, en 1320.

24 Ibid. p. 353, no 32.

25 Ibid. p. 44, no. 83 (Medicina...); p. 49, no. 109 (Scio quod arduum, traictant do medicine et noblesse); p. 53, no. 134 (Secundus Avicena); p. 63, no. 192, un Regime de vivre. - Une Summa magistri Guidonis, p. 60 no. 173, ne paraît pas concerner Gui de Chauliac. 
Et concluons par l'expression de notre reconnaissance. En cet an de disgrâces cruelles, 1940, l'ouvrage d'Arnaldus Villanovanus, par les soins d'un autre Arnaldus, Noviodunensis, revient en photocopie au Pays de Vaud et entre dans la bibliothèque, non plus ducale, mais cantonale et universitaire. Remercions l'ami que nous fêtons aujourd'hui de renouveler, après cinq cents ans, le geste de François de Russin!
Le Mont, mars 1940.
E. Olivier.

\section{Annexe: Liste de régimes de santé du moyen âge, écrits avant 1501.}

Les incunables ont été relevés en suivant Arnold C. Klebs, Incunabula Scientifica et Medica, Short Title list, 1938. V. aussi K. Sudhoff, Deutsche medizinische Inkunabeln, 1908.

On pourra consulter la liste de 28 numéros terminant la thèse, citée ci dessus, note 20 , de Joh.Brinkmann; elle présente malheureusement bien des confusions et des erreurs. Bernhard Milt a offert à ce même 17 mars 1940 un important Beitrag zur Kenntnis der riittelalterlichen Heilkunde am Bodensee und Oberrhein, publié dès lors dans Vierteljahrschrift der naturforsch. Gesellsch. in Zürich, LXXXV, 1940, p. 263-321; nous lui empruntons quelques titres.

Pour le surplus, nous renvoyons à L. Choulant, Bücherkunde für die ältere Medicin, 1841, au Dictionnaire cité de E. Wickersheimer, et aux ouvrages traitant de l'histoire de la médecine en général, par exemple M. Neuburger, Geschichte der Medizin, II, 1911; MeyerSteinegg et Sudhoff, Geschichte der Medizin, 3e éd. 1928; A. Castiglioni, Histoire de la médecine, éd. française 1931.

Ont été portés sur notre liste seulement les régimes embrassant l'ensemble de l'hygiène. Ceux qui s'adressent spécialement aux divers âges, à certaines classes professionnelles, les régimes pour temps de peste ou d'épidémie, ont été laissés de côté.

L'ordre adopté est chronologique, d'après l'époque, non de la publication des écrits, mais de leur rédaction; pour autant qu'elle est connue.

Ont été imprimés au 15e siècle (d'après Klebs)

1. Epistola Aristotelis ad Alexandrum de observatione diaetae. Constitue une partie des Secreta Secretorum, traduits de l'arabe par Philippe de Tripoli. La lettre à Aristote, traduite à son tout d'arabe en latin, à Tolède, par le Juif baptisé Jean, dit de Tolède; entre 1125 et 1150 .

Klebs, 96.1-96.007, s.v. Aristotle; en particulier 96.01 (dès 1472). - Meyer-Steinegg et Sudhoff, 204. - Neuburger II 332. - Wickersheimer 493, s. v. Jean de Toleto. J. Brinkmann.

2. Mö̈se Maimonide, de Cordoue, 1139-1208, élève d'Averroès, deviendra médecin de Saladin, pour lequel il écrit en arabe: Tractatus de regimine sanitatis. Nombreuses trad. en hébreu, latin, allemand. La tombe de M., à Tibériade, est encore lieu de pélerinage. Klebs 643.1 (vers 1481). - Choulant 378. - Neuburger II 222. - Castiglioni 237. 
3. Aldebrandin (Alebrans, etc.) de Sienne, $\dagger$ à Troyes vers 1287, écrit en français, probablement vers 1256 et en hommage à Béatrice de Savoie comtesse de Provence, le Régime du corps.

Klebs 42.1 (1481). - Edition critique par Landouzy et Pépin, 1911. - Wickersheimer 17. - Castiglioni 297.

4. Guillaume de Saliceto, de Plaisance, $\dagger$ vers 1280 ; si toutefois c'est bien l'illustre chirurgien qui a écrit aussi: De Salute corporis.

Klebs 484.1-5 (1472--1500). - Neuburger II 381 ss.

5. Taddeo Alderotti (env. 1223-1303), de Florence, à Bologne où il est professeur dès 1260; né dans la misère, amassera une énorme fortune. Le texte latin de son Libellus de conservanda sanitate, ou de regimine sanitatis secundum quatuor anni tempora, a été imprimé en 1477, avec notre no 13: Klebs 174.2. Une version italienne Libello per conservare la sanita del corpo, connue de Dante, qui avait suivi les cours de Taddeo, fut vivement critiquée par lui pour son mauvais style. Elle a été publiée pour la lère fois par Puccinotti au 19e siècle, d'après Castiglioni 278, tandis que Neuburger II 373 ss. en indique une éd. 1477. - Cf. Wickersheimer 748 Thadée de Parisio.

6. Arnauld de Villeneuve, $\dagger$ décembre 1311. Regimen sanitatis, ad inclitum regem Aragonum directum et ordinatum; écrit aux environs de 1300?. C'est l'ouvrage traduit par Héronchel.

Klebs 99.1 (1474) et 829.1-6 (1480 ss.). - Hauréau et Wickersheimer, ouvr. cités. Neuburger II 391.

7. Regimen sanitatis Salernitanum.

Klebs 829.1-832.11 (1480-1500). Et innombrables éditions ultérieures, traductions et remaniements. - Sudhoff pense qu'Arnauld pourrait bien avoir été plus que le commentateur de ce poème; v. Wickersheimer, 47-48.

8. Magnino, Maynus de Mayneriis, de Milan, maître régent de Paris 1326, à Milan, Venise, etc., encore vivant 1364. Médecin et clerc, marié. Son Regimen sanitatis est de 1331 à 1333. Plus tard, il a été à tort attribué à Arnauld de Villeneuve et publié avec les œuvres de celui-ci.

Klebs 640.1-6, dès 1482. - Neuburger II 391. - Wickersheimer 533. - Cf. no 18.

9. Ugo Benzi, Senensis, Hugues B., de Sienne, Dr de Pavie 1396, professeur, † 1439. Trattato utilissimo circa lo regimento et la conservatione della sanitade. Ecrit seulement en langue vulgaire.

Klebs 1000.1, en 1481; réédité à diverses reprises au siècle suivant. - Wickersheimer 303 (Hugues Benzi). - Neuburger II 506.- Castiglioni 297.

10. Sigismundus Albicus, Sigmund Albich, de Moravie, 1347-1427; étudie à Prague où il professe dès env. 1381; 1411 archevêque de Prague; chassé par les Hussites il mourra en Hongrie où il s'était réfugié. Son Tractatus de regimine hominis reçut ensuite aussi le nom de Vetularius.

Klebs 34.1 (1484). - Neuburger II, 504 s.

11. Anonyme, Regimen sanitatis, zu deutsch Ordnung der Gesuntheit; malgré le titre mi latin, texte uniquement allemand. Dédié dem Grafen von Hochenburg und seiner Gemahlin Margarethe von Thierstein.

Klebs 828.1-10, dès 1472. - Sudhoff, Inkunabeln p. 8 ss. - B. Milt, p. 293, a identifié le comte de Hohenburg, jusque là inconnu; il est attesté entre 1393 et 1432. 
12. Heinrich Louffenberg, de Laufenburg sur le Rhin, $\dagger$ à Strasbourg 1458, achève en 1429 son poème, Versehung des Leibs; arrangement du Régime de Salerne.

Klebs 616.1, serait de 1491. - Pour Sudhoff, Inkunabeln, p. 178 ss., de 1481, avec d'abondantes et intéressantes illustrations. - B. Milt, 289.

13. Benedictus de Nursia, professeur à Pérouse, seconde moitié du $15 \mathrm{e}$ siècle, médecin du pape Sixte IV (1471-84); (ne pas confondre avec Saint Benoît, 480-543, fondateur du Mont Cassin). De conservatione sanitatis.

Klebs 174.1-7, de 1475 à 1483. - Neuburger II 443, 512. - Une note de Klebs, janv. 1940, le résume ainsi: 96 chap.; 1-5 de aere, de exercitio et quiete; de somno et vigilia; de purgandis; de cibo et potu. Ensuite, .. de caseo, de fungis, de lacte, de melle, pisces, de pane..; de animae passionibus. - Je ne sais comment accorder cela avec l'indication du titre donné par le Catal. des Sc. médicales, Bibl. Nat. Paris, 1857, I, p. 444, cote $\mathrm{Tc}^{8}, 1$ : Libellus de conservatione sanitatis, secundum ordinem alphabeti distinctus; dans cette éd., s.l.n.d. la matière serait distribuée comme dans un dictionnaire?

14. Marsilius Ficinus, de Florence (1433-1499). La seconde partie (de vita producenda) de son De triplici vita, est un régime de santé.

Klebs, 397.1-7; 1489 etc.

15. Burckhard von Horneck, Carmen de ingenio sanitatis.

Klebs 231.1, 1500. J'ignore tout de l'auteur, du temps où il a vécu, et de son livre.

Je ne sais davantage que faire du no 21 de Brinkmann, qu'il présente comme un incunable:

B. de Riguartadis, De conservatione sanitatis, Rome 1490, et qui ne figure pas sous ce nom dans Klebs.

Sont restés manuscrits

16. Pierre d'Espagne, le futur pape Jean XXI; vers 1260-70.

a) De conservanda sanitate; mss. à Colmar, Leipzig, Brit. Mus.; de 1 à 4 ff.

b) Regimen sanitatis; B.N. Paris; serait dédié à Blanche de Castille, reine de France;

c) Epistola ad imperatorem Fridericum super regimine sanitatis; Brit. Mus., 3 ff.

Outre une Dieta in cirurgicis et un Regimen salutis per omnes menses, qui sortiraient de notre cadre.

17. Arnauld de Villeneuve. - De conservatione humane vite. Munich, lat. 25.000, f.86s. Wickersheimer 48.

18. Magnino, Maynus de Mayneriis. - Alors que son Regimen, no 8 ci-dessus, dédié à André Ghini de Malpighi, évêque d'Arras, a été imprimé à plusieurs reprises avant 1501 déjà, un autre Regimen sanitatis de lui, dédié à Antonio Fieschi, de Flisco, en 22 chapitres, est resté inédit. Bibl. de l'Arsenal, Paris. - Wickersheimer 533.

19.-20. Conrad Heingartner, de Zurich, licencié en phil., puis (1466) en méd. de Paris, encore vivant 1488, médecin du duc Jean II de Bourbon, au service de Louis XI et de Charles VIII, iatromathématicien, astrologue, le plus savant médecin suisse du 15e siècle. Il écrit, 1477, pour le duc de Bourbon un Regimen sanitatis (à la Bibl. Nat. à Paris) et en 1480, pour la duchesse, un Liber de morborum causis tam coelestibus quam terrenis, où la partie astrologique est complétée par un régime de vivre détaillé, $D e$ 
sanitatis comparandae mediis. (Bibl. Zurich). - B. Milt, 298-303 avec bibliographie. Wickersheimer 107.

21. Conrad Türst, aussi de Zurich et élève de Heingartner; étudie à Paris, Dr en médecine. Né vers 1450-60, exercera à Berne, puis à Zurich où il est depuis 1489 médecin de la ville, passe 1497 à Innsbruck et $\dagger$ vers 1503. En 1482 écrit pour Rodolphe d'Erlach une consultation, dont le second chapitre est un régime de santé. - B. Milt, 305-307, avec bibliogr.

\section{Publiés à partir de 1501}

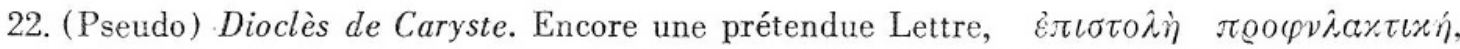
Dioclis ad Antigonum regem de tuenda valetudine epistola. lère impression du texte latin, Bâle 1541. - Choulant, 42.

23. Magister Theodorus, méd. de Frédéric II vers 1235, l'empereur lui demande de conservanda sanitate regulas certas. Théodore répond que ce travail n'a plus à être fait, grâce à l'antique Lettre d'Aristote à Alexandre, no 1 . Il résume pourtant ses vues personnelles en brefs conseils judicieux et pratiques. - Sudhoff, Ein diätetischer Brief an Kaiser Friedrich II von seinem Hofphilosophen Magister Theodorus; Arch. für Gesch. der Med. IX, 1915, p. 1-9.

24. Pierre de Tussignan. Tractatus de regimine sanitatis, ed. per insignem virum P. de Tuxigano, medicinae monarcham, Doctorem Bononiae ... D'après ce titre, emprunté au Cat. des Sc. médic., Bibl. Nat. Paris, 1857, I 451, d'un ex. imprimé à Paris 1535, 80 (cote Tc11, 26), il s'agirait du professeur de Bologne, $†$ 1410. Mais Neuburger, II 485, montre qu'il a dû y avoir plusieurs médecins de ce nom, et que l'auteur de ce Régime ayant été maître de Guillaume de Salicet doit être de la première moitié du 13e siècle.

25. (Conradus), (Mainauer Naturlehre), écrite en latin en 1293 par un clerc de St Gall. Publiée par Wackernagel en 1851. A côté d'une partie astronomique, contient un régime de santé, dérivé des Secreta secretorum, soit Lettre d'Aristote, no. 1. - B. Milt, 277 s., avec bibliographie.

26. Bernard de Gordon, professeur à Montpellier, écrit entre 1294 et 1308 Tractatus de conservatione vitae humanae a die nativitatis usque ad ultimam horam mortis. lère éd. Leipzig 1570, 80. - Se compose de 4 parties, souvent considérées comme des ouvrages indépendants. La 4e, de regimine sanitatis. - Wickersheimer, 75.

27. Arnauld de Villeneuve, dans les dernières années de sa vie, entre 1309 et 1311, dédie à Robert, roi de Naples, de Sicile et de Jérusalem, De conservanda juventute et retardanda senectute; imprimé dans les Oeurres complètes, 1504, 1505 et à part en 1511; etc. Trad. italienne 1550, et anglaise manuscrite. - Hauréau, op. cit., p. $56 \mathrm{~s}$.

28. Vital du Four, Joh. Vitalis de Furno, lecteur à Montpellier 1295, franciscain, cardinal, évêque, $\dagger 1327$ à Avignon. - Pro conservanda sanitate ... ad totius humani corporis morbos ... salutarium remediorum ... liber utilissimus, lère éd. Mayence 1531, folio. On a contesté que cet ouvrage fût du cardinal. Neuburger II 357. - Wickersheimer 776.

29. Jaques Albini, de Moncalieri, attesté de 1324 à 134.9, méd. de sa ville natale et de divers princes de Savoie; écrit vers 1340 pour Jaques de Savoie-Achaïe, De sanitatis custodia. Publié en 1906 par G. Carbonelli: Il de sanitatis custodia de Maestro Giacomo Albini di Moncalieri; dans Biblioteca della Societa storica subalpina, XXXV. Wickersheimer 320 . 
30. Magister Gallus semble avoir enseigné l'astronomie et la médecine à l'université de Prague vers 1350-60; fut probablement le médecin de Charles IV, auquel il dédie un Vitae vivendae ratio, qui sera publiée à Prague en 1819 par Fr. Muller. A aussi écrit sur la peste, et sur les urines un traité qui aurait été imprimé au $15 \mathrm{e}$ siècle, mais n'est pas dans Klebs. - Neuburger II, 504.

31. Eustache Deschamps, env. 1346-1407, n'est pas médecin; bourgeois cossu, occupant de hautes charges à la cour de Charles V, il rime abondamment sur toutes gens et choses de son temps. Ami des larges «buveries» et des plats chargés de bons morceaux, - qui lui vaudront la goutte, - il y va néanmoins de son régime de santé: D'un notable enseignement pour continuer santé au corps d'omme, 226 vers débutant par: Pour vostre santé maintenir. Ce sont les habituelles règles de vie modérée, de la Lettre d'Aristote, du Régime de Salerne, d'Arnauld, sans rien de personnel. Publié en 1893, Oeuvres complètes, VIII, 339-346.

32. Maestro Gregorio (14e siècle), Fiori di medicina; p. p. Franc. Zambrini dans Scelte di curiosita letterarie. Bologne 1859. - Brinkmann, no. 28.

33. Heinricus de Wittenwil (vers 1430) du Toggenbourg, advocatus curiae, introduit dans son poème, Der Ring, un bref régime de santé; ses éléments se rattachant tantôt à Salerne, tantôt à la Lettre d'Aristote, tantôt à Avicenne. Publié 1931. - B. Milt, 290-293, avec bibliogr.

34. (Anonyme, époque pas indiquée). Ein altneapolitanisches Regimen Sanitatis; p. p. Adolf Mussafia, Sitzungsberichte der phil.-hist. Klasse der Kais. Akademie der Wissenschaften, Bd. 100, Mitteilungen aus romanischen Handschriften, Teil I; Vienne 1884. - Brinkmann, no. 18.

35. (Anonyme, début du 16e siècle) Regimen sanitatis (Vademecum of the famous Macheaths physicians); p. p. Cameron Gillies, Glasgow 1912. - Brinkmann, no. 9.

36. Bien que sa lère éd. soit de 1514, nous pouvons enfin accueillir dans notre liste la Rosa Gallica de Symphorien Champier (1471-1538); sur laquelle voir P. Allut, Symphorien Champier, 1859, p. 167-171. Ses sept livres couvrent le même domaine que les régimes de santé; 135 ff. $8^{\circ}: \ldots$ praecepta (empruntés à une dizaine d'auteurs nommés dans le titre, d'Hippocrate à Avicenne, et plusieurs autres) ... quae ad medicam artem rectamque vivendi formam plurimum conducunt ... - Wickersheimer $745 \mathrm{~s}$.

E. Olivier.

\section{Eine Vorlesung über den Kaisersdhnitt aus dem Anfang des I9. Jahrhunderts}

Bekanntlich war der Kaiserschnitt in der vorantiseptischen Zeit eine gefährliche und gefürchtete Operation. Dennoch konnte er nicht immer umgangen werden, wenn es galt, bei besonderen Geburtsfällen, wo die Geburt auf den natürlichen Wegen unmöglich war, Mutter und Kind womöglich zu retten. 